CRYSTALLOGRAPHIC COMMUNICATIONS

ISSN 2056-9890

Received 31 May 2015

Accepted 17 June 2015

Edited by A. J. Lough, University of Toronto, Canada

Keywords: crystal structure; conformation; dihydropyrimidine; ring motif; $\mathrm{C}-\mathrm{H} \cdots \pi$ interactions

CCDC reference: 1407186

Supporting information: this article has

supporting information at journals.iucr.org/e

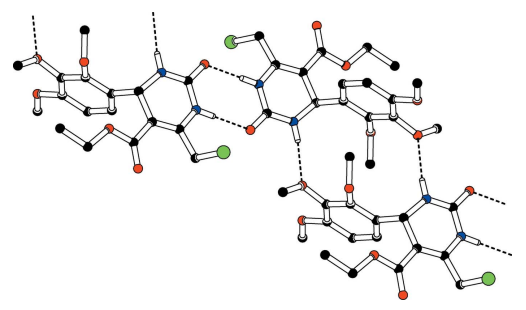

OPEN $\odot$ ACCESS

\section{Crystal structure of ethyl 6-chloromethyl-2-oxo-4- (2,3,4-trimethoxyphenyl)-1,2,3,4-tetrahydro- pyrimidine-5-carboxylate}

\author{
M. Suresh, ${ }^{a}$ M. Syed Ali Padusha, ${ }^{a}$ J. Josephine Novina, ${ }^{b}$ G. Vasuki, ${ }^{c} *$ Vijayan \\ Viswanathan ${ }^{\mathrm{d}}$ and Devadasan Velmurugan ${ }^{\mathrm{d}}$
}

apG \& Research Department of Chemistry, Jamal Mohamed College (Autonomous), Tiruchirappalli-20, India, ${ }^{\mathbf{b}}$ Department of Physics, Idhaya College for Women, Kumbakonam-1, India, ' Department of Physics, Kunthavai Naachiar Government Arts College (W) (Autonomous), Thanjavur-7, India, and ${ }^{\mathbf{d}}$ Centre of Advanced Study in Crystallography and Biophysics, University of Madras, Guindy Campus, Chennai-25, India. *Correspondence e-mail:

vasuki.arasi@yahoo.com

In the title compound, $\mathrm{C}_{17} \mathrm{H}_{21} \mathrm{ClN}_{2} \mathrm{O}_{6}$, the dihydropyrimidine ring adopts a flattened envelope conformation, with the $s p^{3}$-hybridized $\mathrm{C}$ atom forming the flap. The dihedral angle between the least-squares planes of the benzene and dihydropyrimidine rings is $88.09(6)^{\circ}$. An Intramolecular $\mathrm{C}-\mathrm{H} \cdots \mathrm{O}$ hydrogen bond generates an $S(6)$ ring. In the crystal, molecules are linked via pairs of $\mathrm{N}-$ H. . O hydrogen bonds, forming inversion dimers with an $R_{2}^{2}(8)$ ring motif, and the dimers are linked via further pairs of $\mathrm{N}-\mathrm{H} \cdots \mathrm{O}$ hydrogen bonds, forming $R_{2}^{2}(14)$ rings and chains of molecules along [111]. Pairs of inversion-related chains are linked via weak $\mathrm{C}-\mathrm{H} \cdots \pi$ interactions.

\section{Chemical context}

Pyrimidine derivatives have been investigated extensively due to their great biological significance and as the main constituent of nucleic acids. Pyrimidines and their derivatives are considered to be important for drugs and agricultural chemicals. They are also found to exhibit remarkable pharmacological activities such as anti-cancer, anti-tumor, antiinflammatory and antifungal etc and are used widely as agrochemicals, pharmaceuticals, dyes, organic additives in electroplating of steel and in the polymerization process (Sharma et al., 2014; Vaisalini et al., 2012). Dihydropyrimidinones, the product of the Biginelli reaction, are also widely used in the pharmaceutical industry as calcium channel blockers and alpha-1 antagonists (Beena \& Akelesh, 2012). Moreover, some bioactive alkaloids such as batzelladine B, containing the dihydropyrimidine unit, which has been isolated from marine sources, show anti-HIV activity (Asghari et al., 2011). Our interest in the preparation of pharmacologically active compounds led us to synthesize the title compound (I) and we report its crystal structure herein.

\section{Structural commentary}

The molecular structure of (I) is shown in Fig. 1. The dihydropyrimidine ring adopts a flattened envelope conformation. Atoms N1/N2/C11/C12/C14 are essentially planar with a maximum deviation of 0.0305 (17) $\AA$ for $\mathrm{C} 11$ while atom $\mathrm{C} 13$ is displaced by 0.1311 (17) $\AA$ from this plane, forming the flap. The puckering parameters are $q 2=0.0935, q 3=-0.0317, Q=$ $0.0987 \AA, \Theta=108.7$ and $\Phi=22.9^{\circ}$. The benzene ring is almost 
perpendicular to the least-squares plane of the six-membered tetrahydropyrimidine ring, making a dihedral angle of $88.09(6)^{\circ}$.<smiles>CCOC(=O)C1=C(CCl)NC(=O)NC1c1ccc(OC)c(OC)c1OC</smiles>

In comparison, this dihedral angle in the structure of ethyl 6-ethoxycarbonylmethyl-4-(2-hydroxyphenyl)-2-oxo-1,2,3,4tetrahydropyrimidine-5-carboxylate, (II), is 87.7 (2) (Kettmann et al., 2008), in ethyl-6-(chloromethyl)-4-(4-chlorophenyl)-2-oxo-1,2,3,4-tetrahydropyrimidine-5-carboxylate, (III), it is $87.08(9)^{\circ}$ (Bharanidharan et al., 2014), and in the crystal structure of ethyl 6-methyl-2-oxo-4-(3,4,5-trimethoxyphenyl)-1,2,3,4-tetrahydropyrimidine-5-carboxylate, (IV), it is $75.25(6)^{\circ}$ (Novina et al., 2015). The ethyl acetate group attached to the pyrimidine ring shows an extended conformation [torsion angle $\mathrm{C} 12-\mathrm{C} 15-\mathrm{O} 2-\mathrm{C} 16=-175.83(15)^{\circ}$ ]. The methoxy group at $\mathrm{C} 4$ is essentially coplanar with the benzene ring $\left[\mathrm{C} 5-\mathrm{C} 4-\mathrm{O} 5-\mathrm{C} 7=-1.3(3)^{\circ}\right]$, whereas the two methoxy substituent groups at $\mathrm{C} 2$ and $\mathrm{C} 3$ deviate significantly from the benzene plane $[\mathrm{C} 3-\mathrm{C} 2-\mathrm{O} 3-\mathrm{C} 9=71.6$ (2) and $\left.\mathrm{C} 2-\mathrm{C} 3-\mathrm{O} 4-\mathrm{C} 8=71.6(2)^{\circ}\right]$. The molecular structure is partially stabilized by the $\mathrm{C} 10-\mathrm{H} 10 A \cdots \mathrm{O} 1$ intramolecular interaction (Table 1), which generates an $S(6)$ ring motif.

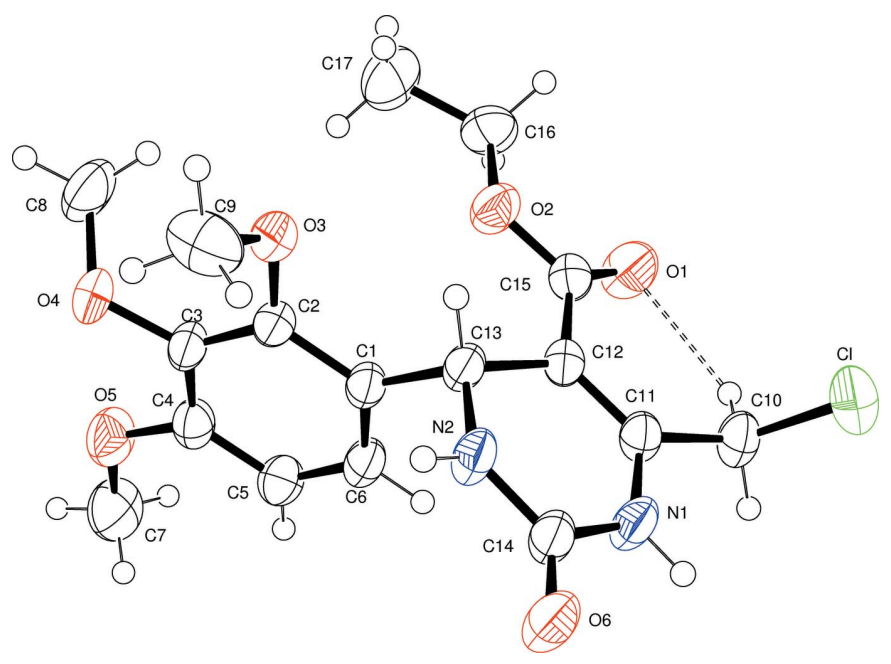

Figure 1

The molecular structure of the title compound, with displacement ellipsoids drawn at the $50 \%$ probability level. The dashed line indicates the intramolecular $\mathrm{C} 10-\mathrm{H} 10 A \cdots \mathrm{O} 1$ hydrogen bond.
Table 1

Hydrogen-bond geometry $\left(\AA{ }^{\circ}\right)$.

$C g$ is the centroid of the $\mathrm{N} 1 / \mathrm{C} 11-\mathrm{C} 13 / \mathrm{N} 2 / \mathrm{C} 14$ pyrimidine ring.

\begin{tabular}{|c|c|c|c|c|}
\hline$D-\mathrm{H} \cdots A$ & $D-\mathrm{H}$ & $\mathrm{H} \cdots A$ & $D \cdots A$ & $D-\mathrm{H} \cdots A$ \\
\hline $\mathrm{N} 1-\mathrm{H} 1 N \cdots \mathrm{O} 6^{\mathrm{i}}$ & 0.86 & 1.95 & $2.812(2)$ & 178 \\
\hline $\mathrm{N} 2-\mathrm{H} 2 N \cdots \mathrm{O} 4^{\mathrm{ii}}$ & 0.86 & 2.37 & $3.160(2)$ & 153 \\
\hline $\mathrm{C} 17-\mathrm{H} 17 C \cdots C g^{\mathrm{iii}}$ & 0.96 & 2.83 & $3.676(4)$ & 147 \\
\hline $\mathrm{C} 10-\mathrm{H} 10 A \cdots \mathrm{O} 1$ & 0.97 & 2.14 & $2.864(3)$ & 131 \\
\hline
\end{tabular}

\section{Supramolecular features}

In the crystal, both $\mathrm{N}-\mathrm{H}$ groups participate in intermolecular hydrogen-bonding associations (Table 1) giving centrosymmetric cyclic motifs [graph sets $R_{2}^{2}(8)$ and $R_{2}^{2}(14)$ ], resulting in ribbons parallel to [111] (Fig. 2). The packing (Fig. 3) also

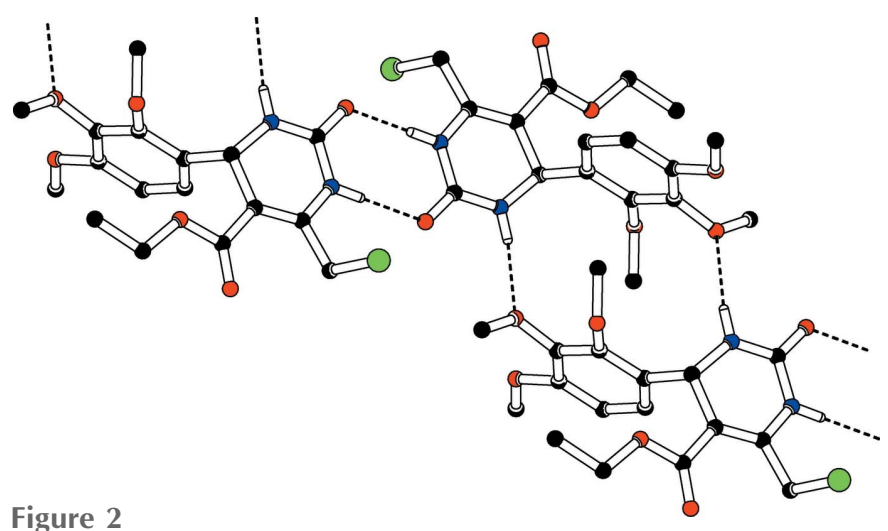

Partial crystal packing diagram for the title compound, showing the $R_{2}^{2}(8)$ and $R_{2}^{2}(14)$ ring motifs. Hydrogen bonds are shown as dashed lines.

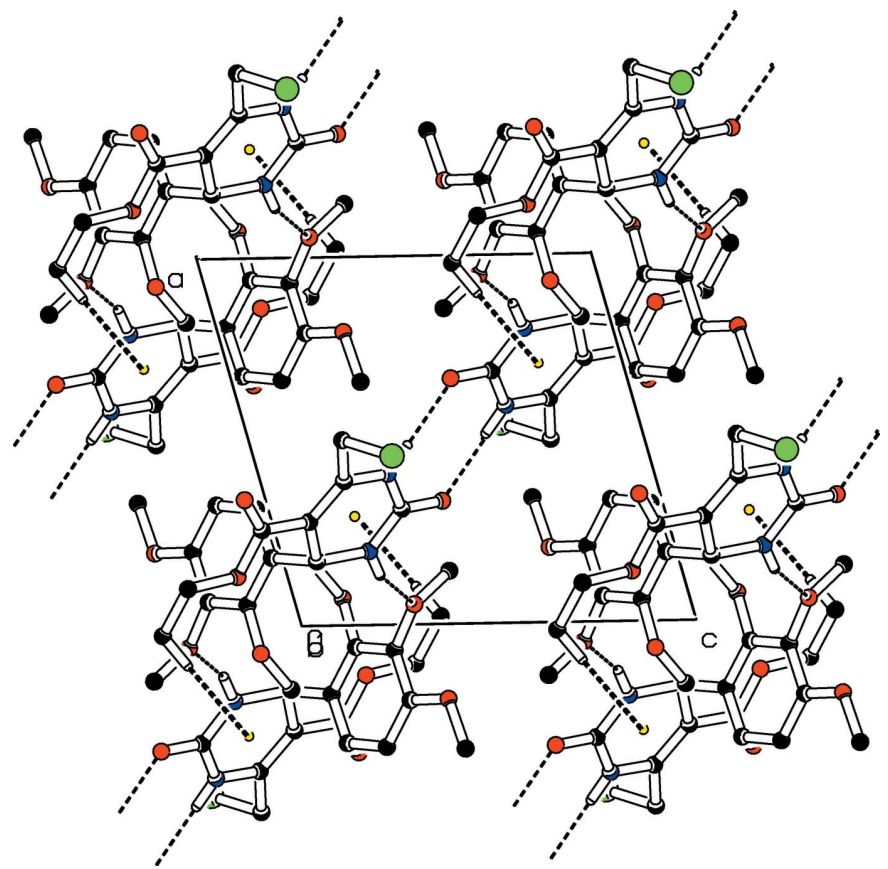

Figure 3

Part of the crystal packing of the title compound, showing $\mathrm{C}-\mathrm{H} \cdots \pi$ interactions and $\mathrm{N}-\mathrm{H} \cdots \mathrm{O}$ hydrogen bonds as dashed lines. 
Table 2

Experimental details.

\begin{tabular}{|c|c|}
\hline \multicolumn{2}{|l|}{ Crystal data } \\
\hline Chemical formula & $\mathrm{C}_{17} \mathrm{H}_{21} \mathrm{ClN}_{2} \mathrm{O}_{6}$ \\
\hline$M_{\mathrm{r}}$ & 384.81 \\
\hline Crystal system, space group & Triclinic, $P \overline{1}$ \\
\hline Temperature $(\mathrm{K})$ & 293 \\
\hline$a, b, c(\AA)$ & $9.479(5), 10.080(5), 10.320(5)$ \\
\hline$\alpha, \beta, \gamma\left({ }^{\circ}\right)$ & $108.552(5), 102.886(5), 94.406(5)$ \\
\hline$V\left(\AA^{3}\right)$ & $899.5(8)$ \\
\hline$Z$ & 2 \\
\hline Radiation type & Mo $K \alpha$ \\
\hline$\mu\left(\mathrm{mm}^{-1}\right)$ & 0.25 \\
\hline Crystal size $(\mathrm{mm})$ & $0.20 \times 0.15 \times 0.10$ \\
\hline \multicolumn{2}{|l|}{ Data collection } \\
\hline Diffractometer & Bruker Kappa APEXII CCD \\
\hline Absorption correction & $\begin{array}{l}\text { Multi-scan ( } S A D A B S \text {; Bruker, } \\
\text { 2008) }\end{array}$ \\
\hline$T_{\min }, T_{\max }$ & $0.952,0.976$ \\
\hline $\begin{array}{l}\text { No. of measured, independent and } \\
\text { observed }[I>2 \sigma(I)] \text { reflections }\end{array}$ & $12878,3737,3025$ \\
\hline$R_{\text {int }}$ & 0.025 \\
\hline$(\sin \theta / \lambda)_{\max }\left(\AA^{-1}\right)$ & 0.631 \\
\hline \multicolumn{2}{|l|}{ Refinement } \\
\hline$R\left[F^{2}>2 \sigma\left(F^{2}\right)\right], w R\left(F^{2}\right), S$ & $0.041,0.121,1.04$ \\
\hline No. of reflections & 3737 \\
\hline No. of parameters & 239 \\
\hline $\mathrm{H}$-atom treatment & $\mathrm{H}$-atom parameters constrained \\
\hline$\Delta \rho_{\max }, \Delta \rho_{\min }\left(\mathrm{e} \AA^{-3}\right)$ & $0.25,-0.28$ \\
\hline
\end{tabular}

Computer programs: APEX2, SAINT and XPREP (Bruker, 2008), SIR92 (Altomare et al., 1993), SHELXL97 (Sheldrick, 2008), ORTEP-3 for Windows (Farrugia, 2012) and PLATON (Spek, 2009).

features weak $\mathrm{C}-\mathrm{H} \cdots \pi$ interactions between the methyl $\mathrm{H}$ atoms of the ethyl groups and the pyrimidine rings of inversion-related molecules.

\section{Synthesis and crystallization}

To an ethanolic solution of ethyl 4-chloroaceto acetate $(2 \mathrm{ml}$, $0.012 \mathrm{~mol}), 2,3,4$-trimethoxy benzaldehyde $(2.4 \mathrm{~g}, 0.012 \mathrm{~mol})$, and urea $(2.25 \mathrm{~g}, 0.037 \mathrm{~mol})$ were added followed by $\mathrm{CeCl}_{3} \cdot 7 \mathrm{H}_{2} \mathrm{O}(931 \mathrm{mg})$. The reaction mixture was taken in a round-bottom flask and refluxed for $2 \mathrm{~h}$. Then the reaction mixture was cooled and poured into crushed ice taken in a beaker with constant stirring. The solid separated out was filtered, washed with ice-cold water and then recrystallized from hot ethanol to afford the product [yield: 92\%; m.p. 425$427 \mathrm{~K}$ ] as X-ray quality crystals.

\section{Refinement}

Crystal data, data collection and structure refinement details are summarized in Table 2. $\mathrm{H}$ atoms were placed in geometrically idealized positions and refined as riding on their parent atoms with $\mathrm{C}-\mathrm{H}$ distances fixed in the range $0.93-0.98 \AA$ and $\mathrm{N}-\mathrm{H}=0.86 \AA$ with $U_{\text {iso }}(\mathrm{H})=1.5 U_{\text {eq }}\left(\mathrm{CH}_{3}\right)$ and $1.2 U_{\text {eq }}\left(\mathrm{CH}_{2}, \mathrm{CH}, \mathrm{NH}\right)$.

\section{Acknowledgements}

The authors thank the TBI X-ray facility, CAS in Crystallography and Biophysics, University of Madras, India, for the data collection.

\section{References}

Altomare, A., Cascarano, G., Giacovazzo, C. \& Guagliardi, A. (1993). J. Appl. Cryst. 26, 343-350.

Asghari, S., Tajbakhsh, M., Kenari, B. J. \& Khaksar, S. (2011). Chin. Chem. Lett. 22, 127-130.

Beena, K. P. \& Akelesh, T. (2012). Int. Res. J. Pharm. 3, 303-304.

Bharanidharan, S., Saleem, H., Gunasekaran, B., Padusha, M. S. A. \& Suresh, M. (2014). Acta Cryst. E70, o1185-o1186.

Bruker (2008). APEX2, SADABS, SAINT and XPREP. Bruker AXS Inc., Madison, Wisconsin, USA.

Farrugia, L. J. (2012). J. Appl. Cryst. 45, 849-854.

Kettmann, V., Světlík, J. \& Veizerová, L. (2008). Acta Cryst. E64, o1092.

Novina, J. J., Vasuki, G., Suresh, M. \& Padusha, M. S. A. (2015). Acta Cryst. E71, o206-0207.

Sharma, A., Khare, R., Kumar, V., Gupta, G. K. \& Beniwal, V. (2014). Int. J. Pharm. Pharm. Sci. 6, 171-175.

Sheldrick, G. M. (2008). Acta Cryst. A64, 112-122.

Spek, A. L. (2009). Acta Cryst. D65, 148-155.

Vaisalini, N. B., Rao, N. V., Harika, V. B. M. L., Desu, P. K. \& Nama, S. (2012). Int. J. Pharm. Chem. Res. 1, 2278-8700. 


\section{supporting information}

Acta Cryst. (2015). E71, 821-823 [doi:10.1107/S2056989015011688]

\section{Crystal structure of ethyl 6-chloromethyl-2-oxo-4-(2,3,4-trimethoxy- phenyl)-1,2,3,4-tetrahydropyrimidine-5-carboxylate}

\section{Suresh, M. Syed Ali Padusha, J. Josephine Novina, G. Vasuki, Vijayan Viswanathan and Devadasan Velmurugan}

\section{Computing details}

Data collection: APEX2 (Bruker, 2008); cell refinement: APEX2 and SAINT (Bruker, 2008); data reduction: SAINT and XPREP (Bruker, 2008); program(s) used to solve structure: SIR92 (Altomare et al., 1993); program(s) used to refine structure: SHELXL97 (Sheldrick, 2008); molecular graphics: ORTEP-3 for Windows (Farrugia, 2012); software used to prepare material for publication: PLATON (Spek, 2009).

Ethyl 6-chloromethyl-2-oxo-4-(2,3,4-trimethoxyphenyl)-1,2,3,4-tetrahydropyrimidine-5-carboxylate

Crystal data

$\mathrm{C}_{17} \mathrm{H}_{21} \mathrm{ClN}_{2} \mathrm{O}_{6}$

$M_{r}=384.81$

Triclinic, $P \overline{1}$

Hall symbol: -P 1

$a=9.479(5) \AA$

$b=10.080(5) \AA$

$c=10.320(5) \AA$

$\alpha=108.552(5)^{\circ}$

$\beta=102.886(5)^{\circ}$

$\gamma=94.406(5)^{\circ}$

$V=899.5(8) \AA^{3}$

Data collection

Bruker Kappa APEXII CCD diffractometer

Radiation source: fine-focus sealed tube

Graphite monochromator

$\omega$ and $\varphi$ scan

Absorption correction: multi-scan

(SADABS; Bruker, 2008)

$T_{\min }=0.952, T_{\max }=0.976$

Refinement

Refinement on $F^{2}$

Least-squares matrix: full

$R\left[F^{2}>2 \sigma\left(F^{2}\right)\right]=0.041$

$w R\left(F^{2}\right)=0.121$

$S=1.04$

3737 reflections
$Z=2$

$F(000)=404$

$D_{\mathrm{x}}=1.421 \mathrm{Mg} \mathrm{m}^{-3}$

Mo $K \alpha$ radiation, $\lambda=0.71073 \AA$

Cell parameters from 3737 reflections

$\theta=1.0-26.6^{\circ}$

$\mu=0.25 \mathrm{~mm}^{-1}$

$T=293 \mathrm{~K}$

Block, colourless

$0.20 \times 0.15 \times 0.10 \mathrm{~mm}$

12878 measured reflections

3737 independent reflections

3025 reflections with $I>2 \sigma(I)$

$R_{\text {int }}=0.025$

$\theta_{\max }=26.6^{\circ}, \theta_{\min }=2.2^{\circ}$

$h=-11 \rightarrow 11$

$k=-12 \rightarrow 12$

$l=-12 \rightarrow 13$

239 parameters

0 restraints

Primary atom site location: structure-invariant direct methods

Secondary atom site location: difference Fourier map 
Hydrogen site location: inferred from neighbouring sites

$\mathrm{H}$-atom parameters constrained

$$
\begin{aligned}
& w=1 /\left[\sigma^{2}\left(F_{\mathrm{o}}^{2}\right)+(0.0599 P)^{2}+0.3029 P\right] \\
& \text { where } P=\left(F_{\mathrm{o}}^{2}+2 F_{\mathrm{c}}^{2}\right) / 3 \\
& (\Delta / \sigma)_{\max }<0.001 \\
& \Delta \rho_{\max }=0.25 \mathrm{e} \AA^{-3} \\
& \Delta \rho_{\min }=-0.28 \mathrm{e} \AA^{-3}
\end{aligned}
$$

Special details

Geometry. All e.s.d.'s (except the e.s.d. in the dihedral angle between two 1.s. planes) are estimated using the full covariance matrix. The cell e.s.d.'s are taken into account individually in the estimation of e.s.d.'s in distances, angles and torsion angles; correlations between e.s.d.'s in cell parameters are only used when they are defined by crystal symmetry. An approximate (isotropic) treatment of cell e.s.d.'s is used for estimating e.s.d.'s involving l.s. planes.

Refinement. Refinement of $F^{2}$ against ALL reflections. The weighted $R$-factor $w R$ and goodness of fit $S$ are based on $F^{2}$,

\begin{tabular}{|c|c|c|c|c|}
\hline & $x$ & $y$ & $z$ & $U_{\text {iso }} * / U_{\text {eq }}$ \\
\hline $\mathrm{C} 7$ & $0.3380(3)$ & $-0.2172(2)$ & $-0.3248(3)$ & $0.0695(6)$ \\
\hline H7A & 0.3999 & -0.1501 & -0.3444 & $0.104 *$ \\
\hline H7B & 0.3213 & -0.3077 & -0.3989 & $0.104 *$ \\
\hline $\mathrm{H} 7 \mathrm{C}$ & 0.3848 & -0.2260 & -0.2360 & $0.104 *$ \\
\hline $\mathrm{C} 4$ & $0.2052(2)$ & $-0.03983(17)$ & $-0.22112(17)$ & $0.0417(4)$ \\
\hline $\mathrm{C} 5$ & $0.33170(19)$ & $0.04788(18)$ & $-0.12747(18)$ & $0.0428(4)$ \\
\hline H5 & 0.4230 & 0.0206 & -0.1293 & $0.051 *$ \\
\hline C6 & $0.32077(18)$ & $0.17612(18)$ & $-0.03140(17)$ & $0.0404(4)$ \\
\hline H6 & 0.4058 & 0.2338 & 0.0317 & $0.049 *$ \\
\hline $\mathrm{C} 1$ & $0.18792(17)$ & $0.22104(16)$ & $-0.02627(16)$ & $0.0353(3)$ \\
\hline $\mathrm{C} 2$ & $0.06034(17)$ & $0.13322(17)$ & $-0.12190(17)$ & $0.0375(4)$ \\
\hline C3 & 0.06932 (19) & $0.00217(17)$ & $-0.21729(17)$ & 0.0409 (4) \\
\hline $\mathrm{C} 8$ & $-0.1441(3)$ & $-0.0596(3)$ & $-0.4122(2)$ & $0.0685(6)$ \\
\hline H8A & -0.1575 & 0.0377 & -0.3781 & $0.103 *$ \\
\hline H8B & -0.2377 & -0.1197 & -0.4467 & $0.103 *$ \\
\hline $\mathrm{H} 8 \mathrm{C}$ & -0.0969 & -0.0740 & -0.4877 & $0.103^{*}$ \\
\hline $\mathrm{C} 9$ & -0.1707 (3) & 0.1099 & $-0.0726(3)$ & $0.0690(6)$ \\
\hline H9A & -0.1769 & 0.0092 & -0.1149 & $0.104^{*}$ \\
\hline H9B & -0.2658 & 0.1358 & -0.0970 & $0.104 *$ \\
\hline $\mathrm{H} 9 \mathrm{C}$ & -0.1365 & 0.1373 & 0.0285 & $0.104 *$ \\
\hline $\mathrm{C} 13$ & $0.17802(17)$ & $0.35901(16)$ & $0.08517(16)$ & $0.0360(3)$ \\
\hline H13 & 0.0787 & 0.3807 & 0.0601 & $0.043 *$ \\
\hline $\mathrm{C} 12$ & $0.28624(17)$ & $0.48334(16)$ & $0.09400(16)$ & $0.0357(3)$ \\
\hline $\mathrm{C} 11$ & $0.39561(18)$ & $0.54845(16)$ & $0.21121(17)$ & $0.0376(4)$ \\
\hline $\mathrm{C} 14$ & 0.31732 (19) & $0.40206(18)$ & $0.33668(17)$ & $0.0417(4)$ \\
\hline $\mathrm{C} 10$ & $0.5076(2)$ & $0.67371(18)$ & $0.23650(19)$ & $0.0451(4)$ \\
\hline $\mathrm{H} 10 \mathrm{~A}$ & 0.5094 & 0.6851 & 0.1470 & $0.054 *$ \\
\hline $\mathrm{H} 10 \mathrm{~B}$ & 0.6041 & 0.6582 & 0.2793 & $0.054 *$ \\
\hline C15 & $0.26590(19)$ & $0.52688(17)$ & $-0.03168(17)$ & $0.0391(4)$ \\
\hline $\mathrm{C} 16$ & $0.1095(2)$ & $0.4838(2)$ & $-0.2602(2)$ & $0.0548(5)$ \\
\hline
\end{tabular}
conventional $R$-factors $R$ are based on $F$, with $F$ set to zero for negative $F^{2}$. The threshold expression of $F^{2}>\sigma\left(F^{2}\right)$ is used only for calculating $R$-factors $(\mathrm{gt})$ etc. and is not relevant to the choice of reflections for refinement. $R$-factors based on $F^{2}$ are statistically about twice as large as those based on $F$, and $R$ - factors based on ALL data will be even larger.

Fractional atomic coordinates and isotropic or equivalent isotropic displacement parameters $\left(\AA^{2}\right)$ 
supporting information

$\begin{array}{lllll}\text { H16A } & 0.1940 & 0.4739 & -0.2989 & 0.066^{*} \\ \text { H16B } & 0.0869 & 0.5787 & -0.2469 & 0.066^{*} \\ \text { C17 } & -0.0182(3) & 0.3764(3) & -0.3576(2) & 0.0816(8) \\ \text { H17A } & 0.0067 & 0.2832 & -0.3725 & 0.122^{*} \\ \text { H17B } & -0.0438 & 0.3916 & -0.4466 & 0.122^{*} \\ \text { H17C } & -0.1001 & 0.3852 & -0.3166 & 0.122^{*} \\ \text { N2 } & 0.20306(15) & 0.34077(15) & 0.22426(14) & 0.0414(3) \\ \text { H2N } & 0.1367 & 0.2844 & 0.2341 & 0.050^{*} \\ \text { N1 } & 0.41333(16) & 0.50454(16) & 0.32654(15) & 0.0478(4) \\ \text { H1N } & 0.4894 & 0.5439 & 0.3964 & 0.057^{*} \\ \text { O5 } & 0.20239(16) & -0.16966(14) & -0.31766(15) & 0.0586(4) \\ \text { O4 } & -0.05528(14) & -0.09329(13) & -0.29991(14) & 0.0536(4) \\ \text { O3 } & -0.07186(13) & 0.17960(13) & -0.12314(14) & 0.0471(3) \\ \text { O1 } & 0.34931(16) & 0.60863(16) & -0.05290(15) & 0.0615(4) \\ \text { O2 } & 0.13972(14) & 0.45913(14) & -0.12663(13) & 0.0495(3) \\ \text { O6 } & 0.33671(15) & 0.37184(15) & 0.44510(13) & 0.0595(4) \\ \text { C1 } & 0.46401(7) & 0.83004(5) & 0.35072(6) & 0.06618(19)\end{array}$

Atomic displacement parameters $\left(\AA^{2}\right)$

\begin{tabular}{lllllll}
\hline & $U^{11}$ & $U^{22}$ & $U^{33}$ & $U^{12}$ & $U^{13}$ & $U^{23}$ \\
\hline C7 & $0.0724(15)$ & $0.0561(13)$ & $0.0664(14)$ & $0.0167(11)$ & $0.0202(11)$ & $-0.0003(10)$ \\
C4 & $0.0495(10)$ & $0.0351(8)$ & $0.0351(8)$ & $0.0004(7)$ & $0.0089(7)$ & $0.0082(7)$ \\
C5 & $0.0384(9)$ & $0.0438(9)$ & $0.0440(9)$ & $0.0025(7)$ & $0.0120(7)$ & $0.0123(7)$ \\
C6 & $0.0355(8)$ & $0.0396(8)$ & $0.0382(8)$ & $-0.0058(7)$ & $0.0033(6)$ & $0.0097(7)$ \\
C1 & $0.0371(8)$ & $0.0317(7)$ & $0.0324(7)$ & $-0.0034(6)$ & $0.0034(6)$ & $0.0106(6)$ \\
C2 & $0.0355(8)$ & $0.0346(8)$ & $0.0371(8)$ & $-0.0010(6)$ & $0.0016(6)$ & $0.0119(6)$ \\
C3 & $0.0411(9)$ & $0.0340(8)$ & $0.0369(8)$ & $-0.0066(7)$ & $-0.0003(7)$ & $0.0082(7)$ \\
C8 & $0.0629(13)$ & $0.0710(14)$ & $0.0475(11)$ & $-0.0165(11)$ & $-0.0123(9)$ & $0.0127(10)$ \\
C9 & $0.0669(14)$ & $0.0783(15)$ & $0.0892(17)$ & $0.0250(12)$ & $0.0395(13)$ & $0.0495(14)$ \\
C13 & $0.0343(8)$ & $0.0352(8)$ & $0.0316(7)$ & $-0.0030(6)$ & $0.0020(6)$ & $0.0087(6)$ \\
C12 & $0.0380(8)$ & $0.0294(7)$ & $0.0348(8)$ & $0.0013(6)$ & $0.0059(6)$ & $0.0078(6)$ \\
C11 & $0.0393(9)$ & $0.0320(8)$ & $0.0371(8)$ & $-0.0010(6)$ & $0.0078(7)$ & $0.0089(6)$ \\
C14 & $0.0439(9)$ & $0.0385(8)$ & $0.0351(8)$ & $-0.0059(7)$ & $0.0025(7)$ & $0.0107(7)$ \\
C10 & $0.0469(10)$ & $0.0373(9)$ & $0.0426(9)$ & $-0.0075(7)$ & $0.0076(7)$ & $0.0082(7)$ \\
C15 & $0.0424(9)$ & $0.0337(8)$ & $0.0384(8)$ & $0.0052(7)$ & $0.0088(7)$ & $0.0100(7)$ \\
C16 & $0.0629(12)$ & $0.0611(12)$ & $0.0402(9)$ & $0.0124(10)$ & $0.0058(8)$ & $0.0220(9)$ \\
C17 & $0.0997(19)$ & $0.0735(16)$ & $0.0466(12)$ & $0.0001(14)$ & $-0.0117(12)$ & $0.0114(11)$ \\
N2 & $0.0422(8)$ & $0.0407(7)$ & $0.0328(7)$ & $-0.0115(6)$ & $0.0035(6)$ & $0.0096(6)$ \\
N1 & $0.0463(8)$ & $0.0482(8)$ & $0.0369(7)$ & $-0.0168(7)$ & $-0.0066(6)$ & $0.0160(6)$ \\
O5 & $0.0615(9)$ & $0.0436(7)$ & $0.0531(8)$ & $0.0061(6)$ & $0.0102(6)$ & $-0.0030(6)$ \\
O4 & $0.0483(7)$ & $0.0383(6)$ & $0.0535(7)$ & $-0.0110(5)$ & $-0.0075(6)$ & $0.0066(6)$ \\
O3 & $0.0359(6)$ & $0.0398(6)$ & $0.0581(8)$ & $-0.0001(5)$ & $0.0015(5)$ & $0.0154(6)$ \\
O1 & $0.0604(9)$ & $0.0675(9)$ & $0.0557(8)$ & $-0.0128(7)$ & $0.0036(6)$ & $0.0334(7)$ \\
O2 & $0.0519(7)$ & $0.0511(7)$ & $0.0386(6)$ & $-0.0040(6)$ & $-0.0016(5)$ & $0.0180(5)$ \\
O6 & $0.0628(9)$ & $0.0630(9)$ & $0.0412(7)$ & $-0.0235(7)$ & $-0.0084(6)$ & $0.0250(6)$ \\
C1 & $0.0771(4)$ & $0.0375(3)$ & $0.0716(4)$ & $0.0020(2)$ & $0.0145(3)$ & $0.0072(2)$ \\
& & & & & & \\
\hline & & & & & &
\end{tabular}


Geometric parameters $\left(\AA,{ }^{\circ}\right)$

\begin{tabular}{|c|c|c|c|}
\hline $\mathrm{C} 7-\mathrm{O} 5$ & $1.415(3)$ & $\mathrm{C} 13-\mathrm{N} 2$ & $1.474(2)$ \\
\hline C7-H7A & 0.9600 & $\mathrm{C} 13-\mathrm{C} 12$ & $1.523(2)$ \\
\hline C7-H7B & 0.9600 & $\mathrm{C} 13-\mathrm{H} 13$ & 0.9800 \\
\hline $\mathrm{C} 7-\mathrm{H} 7 \mathrm{C}$ & 0.9600 & $\mathrm{C} 12-\mathrm{C} 11$ & $1.341(2)$ \\
\hline $\mathrm{C} 4-\mathrm{O} 5$ & $1.364(2)$ & $\mathrm{C} 12-\mathrm{C} 15$ & $1.475(2)$ \\
\hline $\mathrm{C} 4-\mathrm{C} 5$ & $1.389(2)$ & $\mathrm{C} 11-\mathrm{N} 1$ & $1.378(2)$ \\
\hline $\mathrm{C} 4-\mathrm{C} 3$ & $1.391(3)$ & $\mathrm{C} 11-\mathrm{C} 10$ & $1.500(2)$ \\
\hline $\mathrm{C} 5-\mathrm{C} 6$ & $1.384(3)$ & $\mathrm{C} 14-\mathrm{O} 6$ & $1.230(2)$ \\
\hline $\mathrm{C} 5-\mathrm{H} 5$ & 0.9300 & $\mathrm{C} 14-\mathrm{N} 2$ & $1.335(2)$ \\
\hline $\mathrm{C} 6-\mathrm{C} 1$ & $1.377(3)$ & $\mathrm{C} 14-\mathrm{N} 1$ & $1.369(2)$ \\
\hline $\mathrm{C} 6-\mathrm{H} 6$ & 0.9300 & $\mathrm{C} 10-\mathrm{Cl}$ & $1.783(2)$ \\
\hline $\mathrm{C} 1-\mathrm{C} 2$ & $1.403(2)$ & $\mathrm{C} 10-\mathrm{H} 10 \mathrm{~A}$ & 0.9700 \\
\hline $\mathrm{C} 1-\mathrm{C} 13$ & $1.522(2)$ & $\mathrm{C} 10-\mathrm{H} 10 \mathrm{~B}$ & 0.9700 \\
\hline $\mathrm{C} 2-\mathrm{O} 3$ & $1.370(2)$ & $\mathrm{C} 15-\mathrm{O} 1$ & $1.202(2)$ \\
\hline $\mathrm{C} 2-\mathrm{C} 3$ & $1.396(2)$ & $\mathrm{C} 15-\mathrm{O} 2$ & $1.335(2)$ \\
\hline $\mathrm{C} 3-\mathrm{O} 4$ & $1.3791(19)$ & $\mathrm{C} 16-\mathrm{O} 2$ & $1.448(2)$ \\
\hline $\mathrm{C} 8-\mathrm{O} 4$ & $1.424(3)$ & $\mathrm{C} 16-\mathrm{C} 17$ & $1.487(3)$ \\
\hline $\mathrm{C} 8-\mathrm{H} 8 \mathrm{~A}$ & 0.9600 & $\mathrm{C} 16-\mathrm{H} 16 \mathrm{~A}$ & 0.9700 \\
\hline $\mathrm{C} 8-\mathrm{H} 8 \mathrm{~B}$ & 0.9600 & C16-H16B & 0.9700 \\
\hline $\mathrm{C} 8-\mathrm{H} 8 \mathrm{C}$ & 0.9600 & C17-H17A & 0.9600 \\
\hline $\mathrm{C} 9-\mathrm{O} 3$ & $1.412(2)$ & C17-H17B & 0.9600 \\
\hline C9-H9A & 0.9600 & $\mathrm{C} 17-\mathrm{H} 17 \mathrm{C}$ & 0.9600 \\
\hline C9-H9B & 0.9600 & $\mathrm{~N} 2-\mathrm{H} 2 \mathrm{~N}$ & 0.8600 \\
\hline $\mathrm{C} 9-\mathrm{H} 9 \mathrm{C}$ & 0.9600 & $\mathrm{~N} 1-\mathrm{H} 1 \mathrm{~N}$ & 0.8600 \\
\hline $\mathrm{O} 5-\mathrm{C} 7-\mathrm{H} 7 \mathrm{~A}$ & 109.5 & $\mathrm{C} 12-\mathrm{C} 13-\mathrm{H} 13$ & 108.2 \\
\hline $\mathrm{O} 5-\mathrm{C} 7-\mathrm{H} 7 \mathrm{~B}$ & 109.5 & $\mathrm{C} 11-\mathrm{C} 12-\mathrm{C} 15$ & $122.12(15)$ \\
\hline $\mathrm{H} 7 \mathrm{~A}-\mathrm{C} 7-\mathrm{H} 7 \mathrm{~B}$ & 109.5 & $\mathrm{C} 11-\mathrm{C} 12-\mathrm{C} 13$ & $120.78(14)$ \\
\hline $\mathrm{O} 5-\mathrm{C} 7-\mathrm{H} 7 \mathrm{C}$ & 109.5 & $\mathrm{C} 15-\mathrm{C} 12-\mathrm{C} 13$ & $117.09(13)$ \\
\hline $\mathrm{H} 7 \mathrm{~A}-\mathrm{C} 7-\mathrm{H} 7 \mathrm{C}$ & 109.5 & $\mathrm{C} 12-\mathrm{C} 11-\mathrm{N} 1$ & $120.98(14)$ \\
\hline $\mathrm{H} 7 \mathrm{~B}-\mathrm{C} 7-\mathrm{H} 7 \mathrm{C}$ & 109.5 & $\mathrm{C} 12-\mathrm{C} 11-\mathrm{C} 10$ & $126.74(15)$ \\
\hline $\mathrm{O} 5-\mathrm{C} 4-\mathrm{C} 5$ & $124.55(17)$ & $\mathrm{N} 1-\mathrm{C} 11-\mathrm{C} 10$ & $112.26(14)$ \\
\hline $\mathrm{O} 5-\mathrm{C} 4-\mathrm{C} 3$ & $115.73(15)$ & $\mathrm{O} 6-\mathrm{C} 14-\mathrm{N} 2$ & $123.26(15)$ \\
\hline $\mathrm{C} 5-\mathrm{C} 4-\mathrm{C} 3$ & $119.71(16)$ & $\mathrm{O} 6-\mathrm{C} 14-\mathrm{N} 1$ & $120.67(14)$ \\
\hline $\mathrm{C} 6-\mathrm{C} 5-\mathrm{C} 4$ & $119.45(17)$ & $\mathrm{N} 2-\mathrm{C} 14-\mathrm{N} 1$ & $116.06(15)$ \\
\hline $\mathrm{C} 6-\mathrm{C} 5-\mathrm{H} 5$ & 120.3 & $\mathrm{C} 11-\mathrm{C} 10-\mathrm{Cl}$ & $109.90(13)$ \\
\hline $\mathrm{C} 4-\mathrm{C} 5-\mathrm{H} 5$ & 120.3 & $\mathrm{C} 11-\mathrm{C} 10-\mathrm{H} 10 \mathrm{~A}$ & 109.7 \\
\hline $\mathrm{C} 1-\mathrm{C} 6-\mathrm{C} 5$ & $122.07(15)$ & $\mathrm{Cl}-\mathrm{C} 10-\mathrm{H} 10 \mathrm{~A}$ & 109.7 \\
\hline $\mathrm{C} 1-\mathrm{C} 6-\mathrm{H} 6$ & 119.0 & $\mathrm{C} 11-\mathrm{C} 10-\mathrm{H} 10 \mathrm{~B}$ & 109.7 \\
\hline $\mathrm{C} 5-\mathrm{C} 6-\mathrm{H} 6$ & 119.0 & $\mathrm{Cl}-\mathrm{C} 10-\mathrm{H} 10 \mathrm{~B}$ & 109.7 \\
\hline $\mathrm{C} 6-\mathrm{C} 1-\mathrm{C} 2$ & $118.47(15)$ & $\mathrm{H} 10 \mathrm{~A}-\mathrm{C} 10-\mathrm{H} 10 \mathrm{~B}$ & 108.2 \\
\hline $\mathrm{C} 6-\mathrm{C} 1-\mathrm{C} 13$ & $121.07(13)$ & $\mathrm{O} 1-\mathrm{C} 15-\mathrm{O} 2$ & $122.11(16)$ \\
\hline $\mathrm{C} 2-\mathrm{C} 1-\mathrm{C} 13$ & $120.39(15)$ & $\mathrm{O} 1-\mathrm{C} 15-\mathrm{C} 12$ & $127.08(15)$ \\
\hline $\mathrm{O} 3-\mathrm{C} 2-\mathrm{C} 3$ & $120.68(14)$ & $\mathrm{O} 2-\mathrm{C} 15-\mathrm{C} 12$ & $110.79(14)$ \\
\hline $\mathrm{O} 3-\mathrm{C} 2-\mathrm{C} 1$ & $119.18(15)$ & $\mathrm{O} 2-\mathrm{C} 16-\mathrm{C} 17$ & $107.07(17)$ \\
\hline $\mathrm{C} 3-\mathrm{C} 2-\mathrm{C} 1$ & $120.11(16)$ & $\mathrm{O} 2-\mathrm{C} 16-\mathrm{H} 16 \mathrm{~A}$ & 110.3 \\
\hline
\end{tabular}




\begin{tabular}{|c|c|}
\hline $\mathrm{O} 4-\mathrm{C} 3-\mathrm{C} 4$ & $118.51(15)$ \\
\hline $\mathrm{O} 4-\mathrm{C} 3-\mathrm{C} 2$ & $121.07(16)$ \\
\hline $\mathrm{C} 4-\mathrm{C} 3-\mathrm{C} 2$ & $120.16(14)$ \\
\hline $\mathrm{O} 4-\mathrm{C} 8-\mathrm{H} 8 \mathrm{~A}$ & 109.5 \\
\hline $\mathrm{O} 4-\mathrm{C} 8-\mathrm{H} 8 \mathrm{~B}$ & 109.5 \\
\hline $\mathrm{H} 8 \mathrm{~A}-\mathrm{C} 8-\mathrm{H} 8 \mathrm{~B}$ & 109.5 \\
\hline $\mathrm{O} 4-\mathrm{C} 8-\mathrm{H} 8 \mathrm{C}$ & 109.5 \\
\hline $\mathrm{H} 8 \mathrm{~A}-\mathrm{C} 8-\mathrm{H} 8 \mathrm{C}$ & 109.5 \\
\hline $\mathrm{H} 8 \mathrm{~B}-\mathrm{C} 8-\mathrm{H} 8 \mathrm{C}$ & 109.5 \\
\hline $\mathrm{O} 3-\mathrm{C} 9-\mathrm{H} 9 \mathrm{~A}$ & 109.5 \\
\hline $\mathrm{O} 3-\mathrm{C} 9-\mathrm{H} 9 \mathrm{~B}$ & 109.5 \\
\hline $\mathrm{H} 9 \mathrm{~A}-\mathrm{C} 9-\mathrm{H} 9 \mathrm{~B}$ & 109.5 \\
\hline $\mathrm{O} 3-\mathrm{C} 9-\mathrm{H} 9 \mathrm{C}$ & 109.5 \\
\hline $\mathrm{H} 9 \mathrm{~A}-\mathrm{C} 9-\mathrm{H} 9 \mathrm{C}$ & 109.5 \\
\hline $\mathrm{H} 9 \mathrm{~B}-\mathrm{C} 9-\mathrm{H} 9 \mathrm{C}$ & 109.5 \\
\hline $\mathrm{N} 2-\mathrm{C} 13-\mathrm{C} 1$ & $109.46(13)$ \\
\hline $\mathrm{N} 2-\mathrm{C} 13-\mathrm{C} 12$ & $109.91(12)$ \\
\hline $\mathrm{C} 1-\mathrm{C} 13-\mathrm{C} 12$ & $112.61(13)$ \\
\hline $\mathrm{N} 2-\mathrm{C} 13-\mathrm{H} 13$ & 108.2 \\
\hline $\mathrm{C} 1-\mathrm{C} 13-\mathrm{H} 13$ & 108.2 \\
\hline $\mathrm{O} 5-\mathrm{C} 4-\mathrm{C} 5-\mathrm{C} 6$ & $-178.30(16)$ \\
\hline $\mathrm{C} 3-\mathrm{C} 4-\mathrm{C} 5-\mathrm{C} 6$ & $0.0(3)$ \\
\hline $\mathrm{C} 4-\mathrm{C} 5-\mathrm{C} 6-\mathrm{C} 1$ & $-0.7(3)$ \\
\hline $\mathrm{C} 5-\mathrm{C} 6-\mathrm{C} 1-\mathrm{C} 2$ & $0.0(2)$ \\
\hline $\mathrm{C} 5-\mathrm{C} 6-\mathrm{C} 1-\mathrm{C} 13$ & $176.89(15)$ \\
\hline $\mathrm{C} 6-\mathrm{C} 1-\mathrm{C} 2-\mathrm{O} 3$ & $-176.62(14)$ \\
\hline $\mathrm{C} 13-\mathrm{C} 1-\mathrm{C} 2-\mathrm{O} 3$ & $6.5(2)$ \\
\hline $\mathrm{C} 6-\mathrm{C} 1-\mathrm{C} 2-\mathrm{C} 3$ & $1.4(2)$ \\
\hline $\mathrm{C} 13-\mathrm{C} 1-\mathrm{C} 2-\mathrm{C} 3$ & $-175.49(14)$ \\
\hline $\mathrm{O} 5-\mathrm{C} 4-\mathrm{C} 3-\mathrm{O} 4$ & $5.7(2)$ \\
\hline $\mathrm{C} 5-\mathrm{C} 4-\mathrm{C} 3-\mathrm{O} 4$ & $-172.75(15)$ \\
\hline $\mathrm{O} 5-\mathrm{C} 4-\mathrm{C} 3-\mathrm{C} 2$ & $179.86(15)$ \\
\hline $\mathrm{C} 5-\mathrm{C} 4-\mathrm{C} 3-\mathrm{C} 2$ & $1.4(3)$ \\
\hline $\mathrm{O} 3-\mathrm{C} 2-\mathrm{C} 3-\mathrm{O} 4$ & $-10.1(2)$ \\
\hline $\mathrm{C} 1-\mathrm{C} 2-\mathrm{C} 3-\mathrm{O} 4$ & $171.86(15)$ \\
\hline $\mathrm{O} 3-\mathrm{C} 2-\mathrm{C} 3-\mathrm{C} 4$ & $175.89(15)$ \\
\hline $\mathrm{C} 1-\mathrm{C} 2-\mathrm{C} 3-\mathrm{C} 4$ & $-2.1(2)$ \\
\hline $\mathrm{C} 6-\mathrm{C} 1-\mathrm{C} 13-\mathrm{N} 2$ & $-72.80(18)$ \\
\hline $\mathrm{C} 2-\mathrm{C} 1-\mathrm{C} 13-\mathrm{N} 2$ & $104.02(16)$ \\
\hline $\mathrm{C} 6-\mathrm{C} 1-\mathrm{C} 13-\mathrm{C} 12$ & $49.8(2)$ \\
\hline $\mathrm{C} 2-\mathrm{C} 1-\mathrm{C} 13-\mathrm{C} 12$ & $-133.40(15)$ \\
\hline $\mathrm{N} 2-\mathrm{C} 13-\mathrm{C} 12-\mathrm{C} 11$ & $7.4(2)$ \\
\hline $\mathrm{C} 1-\mathrm{C} 13-\mathrm{C} 12-\mathrm{C} 11$ & $-114.95(17)$ \\
\hline $\mathrm{N} 2-\mathrm{C} 13-\mathrm{C} 12-\mathrm{C} 15$ & $-173.26(14)$ \\
\hline $\mathrm{C} 1-\mathrm{C} 13-\mathrm{C} 12-\mathrm{C} 15$ & $64.41(19)$ \\
\hline $\mathrm{C} 15-\mathrm{C} 12-\mathrm{C} 11-\mathrm{N} 1$ & $-179.15(15)$ \\
\hline
\end{tabular}

\begin{tabular}{|c|c|}
\hline $\mathrm{C} 17-\mathrm{C} 16-\mathrm{H} 16 \mathrm{~A}$ & 110.3 \\
\hline $\mathrm{O} 2-\mathrm{C} 16-\mathrm{H} 16 \mathrm{~B}$ & 110.3 \\
\hline $\mathrm{C} 17-\mathrm{C} 16-\mathrm{H} 16 \mathrm{~B}$ & 110.3 \\
\hline $\mathrm{H} 16 \mathrm{~A}-\mathrm{C} 16-\mathrm{H} 16 \mathrm{~B}$ & 108.6 \\
\hline $\mathrm{C} 16-\mathrm{C} 17-\mathrm{H} 17 \mathrm{~A}$ & 109.5 \\
\hline $\mathrm{C} 16-\mathrm{C} 17-\mathrm{H} 17 \mathrm{~B}$ & 109.5 \\
\hline $\mathrm{H} 17 \mathrm{~A}-\mathrm{C} 17-\mathrm{H} 17 \mathrm{~B}$ & 109.5 \\
\hline $\mathrm{C} 16-\mathrm{C} 17-\mathrm{H} 17 \mathrm{C}$ & 109.5 \\
\hline $\mathrm{H} 17 \mathrm{~A}-\mathrm{C} 17-\mathrm{H} 17 \mathrm{C}$ & 109.5 \\
\hline $\mathrm{H} 17 \mathrm{~B}-\mathrm{C} 17-\mathrm{H} 17 \mathrm{C}$ & 109.5 \\
\hline $\mathrm{C} 14-\mathrm{N} 2-\mathrm{C} 13$ & $127.23(14)$ \\
\hline $\mathrm{C} 14-\mathrm{N} 2-\mathrm{H} 2 \mathrm{~N}$ & 116.4 \\
\hline $\mathrm{C} 13-\mathrm{N} 2-\mathrm{H} 2 \mathrm{~N}$ & 116.4 \\
\hline $\mathrm{C} 14-\mathrm{N} 1-\mathrm{C} 11$ & $124.02(14)$ \\
\hline $\mathrm{C} 14-\mathrm{N} 1-\mathrm{H} 1 \mathrm{~N}$ & 118.0 \\
\hline $\mathrm{C} 11-\mathrm{N} 1-\mathrm{H} 1 \mathrm{~N}$ & 118.0 \\
\hline $\mathrm{C} 4-\mathrm{O} 5-\mathrm{C} 7$ & $117.72(15)$ \\
\hline $\mathrm{C} 3-\mathrm{O} 4-\mathrm{C} 8$ & $117.15(15)$ \\
\hline $\mathrm{C} 2-\mathrm{O} 3-\mathrm{C} 9$ & $116.65(15)$ \\
\hline $\mathrm{C} 15-\mathrm{O} 2-\mathrm{C} 16$ & $117.05(14)$ \\
\hline $\mathrm{C} 13-\mathrm{C} 12-\mathrm{C} 11-\mathrm{N} 1$ & $0.2(3)$ \\
\hline $\mathrm{C} 15-\mathrm{C} 12-\mathrm{C} 11-\mathrm{C} 10$ & $2.3(3)$ \\
\hline $\mathrm{C} 13-\mathrm{C} 12-\mathrm{C} 11-\mathrm{C} 10$ & $-178.40(16)$ \\
\hline $\mathrm{C} 12-\mathrm{C} 11-\mathrm{C} 10-\mathrm{Cl}$ & $103.68(19)$ \\
\hline $\mathrm{N} 1-\mathrm{C} 11-\mathrm{C} 10-\mathrm{Cl}$ & $-75.01(18)$ \\
\hline $\mathrm{C} 11-\mathrm{C} 12-\mathrm{C} 15-\mathrm{O} 1$ & $9.9(3)$ \\
\hline $\mathrm{C} 13-\mathrm{C} 12-\mathrm{C} 15-\mathrm{O} 1$ & $-169.41(18)$ \\
\hline $\mathrm{C} 11-\mathrm{C} 12-\mathrm{C} 15-\mathrm{O} 2$ & $-171.87(16)$ \\
\hline $\mathrm{C} 13-\mathrm{C} 12-\mathrm{C} 15-\mathrm{O} 2$ & $8.8(2)$ \\
\hline $\mathrm{O} 6-\mathrm{C} 14-\mathrm{N} 2-\mathrm{C} 13$ & $-173.33(17)$ \\
\hline $\mathrm{N} 1-\mathrm{C} 14-\mathrm{N} 2-\mathrm{C} 13$ & $7.7(3)$ \\
\hline $\mathrm{C} 1-\mathrm{C} 13-\mathrm{N} 2-\mathrm{C} 14$ & $112.29(19)$ \\
\hline $\mathrm{C} 12-\mathrm{C} 13-\mathrm{N} 2-\mathrm{C} 14$ & $-11.9(2)$ \\
\hline $\mathrm{O} 6-\mathrm{C} 14-\mathrm{N} 1-\mathrm{C} 11$ & $-177.08(18)$ \\
\hline $\mathrm{N} 2-\mathrm{C} 14-\mathrm{N} 1-\mathrm{C} 11$ & $1.9(3)$ \\
\hline $\mathrm{C} 12-\mathrm{C} 11-\mathrm{N} 1-\mathrm{C} 14$ & $-5.6(3)$ \\
\hline $\mathrm{C} 10-\mathrm{C} 11-\mathrm{N} 1-\mathrm{C} 14$ & $173.14(17)$ \\
\hline $\mathrm{C} 5-\mathrm{C} 4-\mathrm{O} 5-\mathrm{C} 7$ & $-1.3(3)$ \\
\hline $\mathrm{C} 3-\mathrm{C} 4-\mathrm{O} 5-\mathrm{C} 7$ & $-179.67(18)$ \\
\hline $\mathrm{C} 4-\mathrm{C} 3-\mathrm{O} 4-\mathrm{C} 8$ & $-114.3(2)$ \\
\hline $\mathrm{C} 2-\mathrm{C} 3-\mathrm{O} 4-\mathrm{C} 8$ & $71.6(2)$ \\
\hline $\mathrm{C} 3-\mathrm{C} 2-\mathrm{O} 3-\mathrm{C} 9$ & $71.6(2)$ \\
\hline $\mathrm{C} 1-\mathrm{C} 2-\mathrm{O} 3-\mathrm{C} 9$ & $-110.4(2)$ \\
\hline $\mathrm{O} 1-\mathrm{C} 15-\mathrm{O} 2-\mathrm{C} 16$ & $2.5(3)$ \\
\hline $\mathrm{C} 12-\mathrm{C} 15-\mathrm{O} 2-\mathrm{C} 16$ & $-175.83(15)$ \\
\hline $\mathrm{C} 17-\mathrm{C} 16-\mathrm{O} 2-\mathrm{C} 15$ & $168.35(19)$ \\
\hline
\end{tabular}


Hydrogen-bond geometry $\left(\AA,{ }^{\circ}\right)$

$\mathrm{Cg}$ is the centroid of the $\mathrm{N} 1 / \mathrm{C} 11-\mathrm{C} 13 / \mathrm{N} 2 / \mathrm{C} 14$ pyrimidine ring.

\begin{tabular}{lllll}
\hline$D-\mathrm{H}^{\prime} \cdots A$ & $D-\mathrm{H}$ & $\mathrm{H} \cdots A$ & $D \cdots A$ & $D-\mathrm{H} \cdots A$ \\
\hline $\mathrm{N} 1-\mathrm{H} 1 N \cdots \mathrm{O} 6^{\mathrm{i}}$ & 0.86 & 1.95 & $2.812(2)$ & 178 \\
$\mathrm{~N} 2-\mathrm{H} 2 N \cdots \mathrm{O} 4^{\mathrm{ii}}$ & 0.86 & 2.37 & $3.160(2)$ & 153 \\
$\mathrm{C} 17-\mathrm{H} 17 C \cdots C g^{\mathrm{iii}}$ & 0.96 & 2.83 & $3.676(4)$ & 147 \\
$\mathrm{C} 10-\mathrm{H} 10 A \cdots \mathrm{O} 1$ & 0.97 & 2.14 & $2.864(3)$ & 131 \\
\hline
\end{tabular}

Symmetry codes: (i) $-x+1,-y+1,-z+1$; (ii) $-x,-y,-z$; (iii) $-x,-y+1,-z+2$. 